\title{
Investigation of Laser Induced Inhibition and Simulation in Biological Samples
}

\author{
Mohamed M. Fadhali ${ }^{1, *}$, Fadhl A. Saeed ${ }^{2}$, Nahlah M. Hashim ${ }^{1}$, Saktioto Toto ${ }^{3}$, Jalil Ali ${ }^{4}$

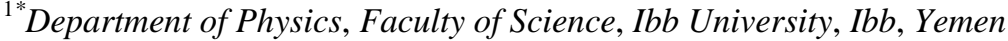 \\ ${ }^{2}$ Department of Microbiology, Faculty of Science, Ibb University, Ibb, Yemen \\ ${ }^{3}$ Physcis Dept. Faculty of Math and Natural Sciences, University of Riau, Indonesia \\ ${ }^{4}$ Advanced Photonics Science Institute, Universiti Teknologi Malaysia, Johor, Malaysia \\ E-mail: "mohamedfadhali@yahoo.com \\ Received May 21, 2011; revised June 24, 2011; accepted July 2, 2011
}

\begin{abstract}
In this research, some experimental measurements have been carried out to study the biological effects induced by laser irradiation on bacterial samples prepared by different ways and at different conditions. Considering the induced samples, the effect of laser irradiation has been investigated through analyzing some of the properties of the transmitted and scattered laser beam for determining the stimulation or inhibition experienced by the investigated sample. In this study absorbance and scattering values have been measured as indicators of sample response to the irradiation laser beam. Absorbance and scattering have been investigated for different irradiation and sample parameters. Significant responses related to inhibition and stimulation effects of the investigated samples have been obtained. These results may significantly contribute in determining the effective utilization of the laser beam as a therapeutic tool for accelerating the wounds and burns healing of diabetic patients whom their response to anti-biotic is not appropriate. The simultaneous irradiation of samples with the use of anti-biotic shows significantly positive effect and fast response.
\end{abstract}

Keywords: Photobiology, Inhibition, Stimulation, Absorption, Laser Therapy

\section{Introduction}

Lasers as highly stable sources of coherent and monochromatic light, have been used extensively in technical applications and for medical therapy. Laser light can interact with tissue in four ways namely: transmission, reflection, scattering and absorption. Transmission refers to the passage of light through a tissue without having any effect on that tissue or on the properties of the light. The transmission of laser radiation in tissues is related to its wavelength. Reflection refers to the repelling of light off the surface of the tissue without entering the tissue. Scattering of light occurs after it has entered the tissue, whereby the beam of light is spread out within the tissue resulting in irradiation of a larger area than anticipated [1-3]. Absorption is a process by which a photon gives up energy to its surrounding medium. This energy is ultimately responsible for photobiostimulation [4,5]. The effect of laser irradiation on biological objects depends on experimental conditions, such as the type of irradiated cells, wavelength and intensity of light, etc. Laser was first used in the medical field as a focused, high power beam with photothermal effects in which tissue was vaporized by the intense heat. It was postulated that surgical lasers normally have Gaussian beam modes. In such mode the laser power is highest at the center of the beam and falling off in a bell-shaped curve with the weakest power at the periphery of the beam diffusing out into the undamaged tissues $[1,6]$. This phenomenon was called the "alpha-phenomenon" [4]. Laser devices were manufactured in which power densities and energy densities of laser were lowered to a point where no photothermal effects occurred but the photo-osmotic, photo-ionic and photo-enzymatic effects were still operative. Applications of lasers are now widespread in almost every medical specialty, especially dermatology, ophthalmology and medical acupuncture [7].

The diverse tissue and cell types in the body all have their own unique light absorption characteristics; that is, they will only absorb light at specific wavelengths and not at others. For example, skin layers, because of their high blood and water content, absorb red light very read- 
ily, while calcium and phosphorus absorb light of a different wavelength [8]. Once a photobiological response is observed, the next step should be to determine the optimum wavelength and dose of radiation to produce the effect, i.e., an action spectrum. An action spectrum is a plot of the relative effectiveness of different wavelengths of light in causing a particular biological response, and under ideal conditions it should mimic the absorption spectrum of the molecule that is absorbing the light, and whose photochemical alteration causes the biological effect. Thus, an action spectrum not only identifies the wavelength(s) that will have the maximum effect with the least dose of radiation, but it also helps to identify the target of the radiation. For example, the action spectrum for killing bacteria mimics the absorption spectrum of deoxyribonucleic acid (DNA) [6,9-11]. This result is understandable in view of the unique importance of DNA to a cell.

Low-level laser Photobiology uses radiation both in the visible $(400 \mathrm{~nm}-700 \mathrm{~nm})$ and in the near-infrared $(700 \mathrm{~nm}-1000 \mathrm{~nm})$ regions of the spectrum. When a photon is absorbed by a molecule, the electrons of that molecule are raised to a higher energy state $[7,8,12]$. This excited molecule must lose its extra energy, and it can do this either by re-emitting a photon of longer wavelength (i.e., lower energy than the absorbed photon) as fluorescence or phosphorescence, or it can lose energy by giving off heat, or it can lose energy by undergoing photochemical alteration.

Photobiological responses are the result of photophysical and/or photochemical changes produced by the absorption of nonionizing radiation. Karu $[6,13]$ has shown that visible and near-infrared radiation is absorbed in the respiratory chain molecules in the mitochondria (e.g., cytochrome), which results in increased metabolism, which leads to signal transduction to other parts of the cell, including cell membranes, and ultimately to the photoresponse (e.g., stimulation of growth) [8,12]. Laser irradiation as a phototherapeutic modality for the induction or acceleration of wound healing was first introduced by Mester et al. $[4,14]$ in the 1970s but still is not an established therapy. This is mainly due to the fact that substantial amounts of research were originally done in East European countries and published in non-peer-reviewed journals. Moreover, there has often been a lack of accuracy in the documentation of exact irradiation protocols and the incorporation of appropriate controls in the past. Additionally, the variety of laser systems and experimental conditions utilized made comparison of results difficult. Since more well-controlled studies have been performed and since the Food and Drug Administration (FDA) has initiated research in the field of low intensity laser therapy [1], this phototherapy is gaining increasing interest.

\section{Experimental Methods}

This research work has been initiated by setting up the experimental set up illustrated in Figure 1 which consists of laser source, sample stage, optical detection circuit and magnetic stirrer.

This experimental setup has been designed for two types of measurements, i.e. transmission and scattering measurements. It consists of a laser, sample holder, magnetic stirrer (to provide a homogeneous distribution of the investigated sample), a photodetection circuit and a display system (Multimeter and digital oscilloscope).

The samples were irradiated with (Diode Pumped Solid State Laser) DPSS of output power of $50 \mathrm{~mW}$ \& $150 \mathrm{~mW}$ and wavelength of $532 \mathrm{~nm}$ (green). The investigated samples were Catalase Enzyme and Staphylococcus bacteria that are prepared with different concentrations using the common biological methods. The experimental results were based on the measurements of the transmitted and/or scattered laser beam at different angles.

\section{Results and Discussions}

\subsection{Results for Catalase Enzyme}

Catalase Enzymes samples are prepared with different concentrations from yeast in cuvette with distilled water and irradiated for different irradiation times. The detection process has been performed using $\mathrm{H}_{2} \mathrm{O}_{2}$. The obtained results revealed that significant effect have been occurred as shown in Figure 2. The reaction time is significantly decreasing with the irradiation time. The reaction time is dependent on the concentration of the sample and increasing with the sample concentration. However, after a specific concentration the reaction time is decreasing with the irradiation time. In this case at a concentration of $0.6 \mathrm{~g} / \mathrm{mL}$ the reaction time is greatest for all irradiation times and drastically decreasing with the in-

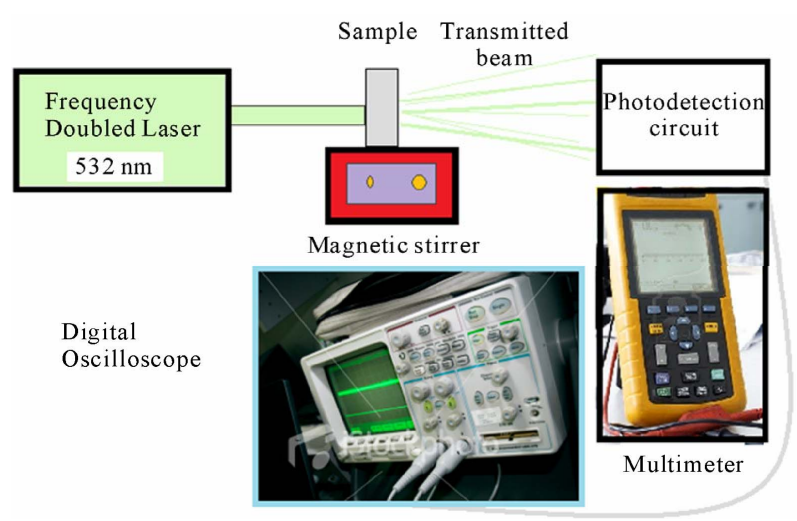

Figure 1. Experimental setup. 


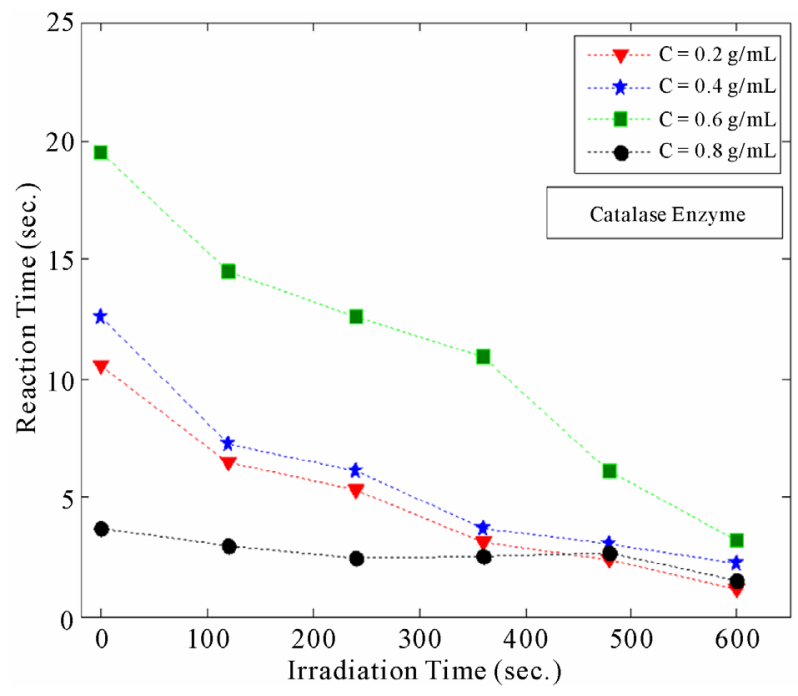

Figure 2. Variation of reaction time with the irradiation time for different sample concentration.

creasing of irradiation time for higher concentration as it is clear for concentration of $0.8 \mathrm{~g} / \mathrm{mL}$ which is depicted in Figure 3. These results assured the applicability of laser irradiation to stimulate Enzymes reaction concentration. It also reveals that there will be an optimum concentration that that exhibit significant response to the laser irradiation. Moreover, the stimulation effect is enhanced with the increasing of irradiation time.

\subsection{Bacteria Sample (Staphylococcus)}

Samples (Staphylococcus injected into normal saline solution) have been clinically collected and prepared in different unit cell of formation (ucf). The samples were put on a stirrer at $15 \mathrm{~cm}$ away from the laser source (laser spot diameter $0.2 \mathrm{~cm}$ ). The samples have been arranged in two different forms, i.e. on plates and/or in cuvettes. The effects of irradiation with laser of $150 \mathrm{~mW}$, $50 \mathrm{~mW}$ and wavelength of $532 \mathrm{~nm}$ on reaction time and absorbance of the prepared samples have been studied for different sample concentration. The obtained results revealed that significant effect have been occurred. The response was also investigated after each experimental procedure using the convenient microbiological analysis and inspections. It has been found that the change in absorbance and/or scattered laser intensity was due to variation of one or more of the characters of bacterial samples, (such as viability or produced enzymes). It is therefore, quite amenable to take the variation in laser absorbance or scattered intensity as indicators to the sample response to the laser irradiation. As shown in Figure 4, the absorbance of the laser beam is found to be strongly dependent on the concentration of the sample and time of irradiation. The inhibition effect was observed with in-

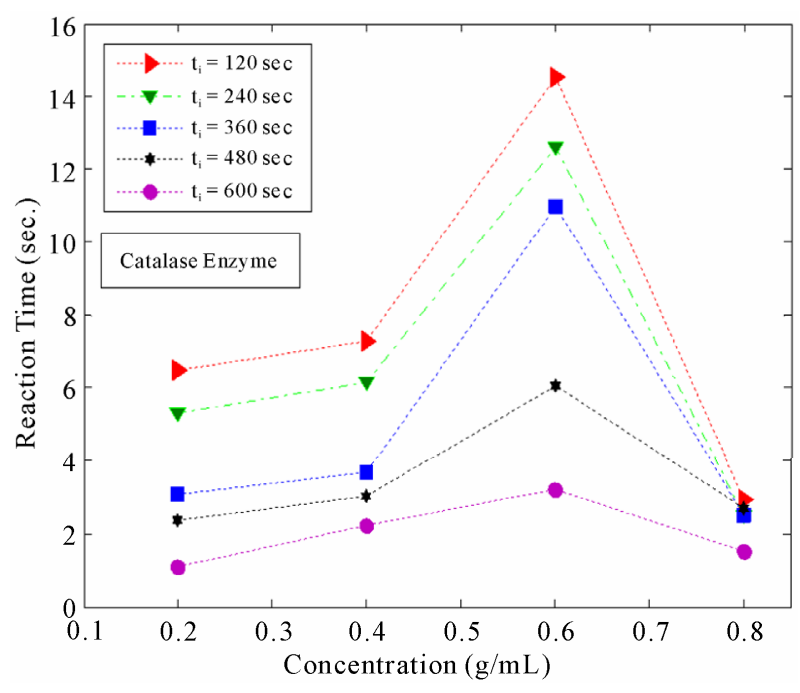

Figure 3. Variation of reaction time with the sample concentration for different irradiation times.

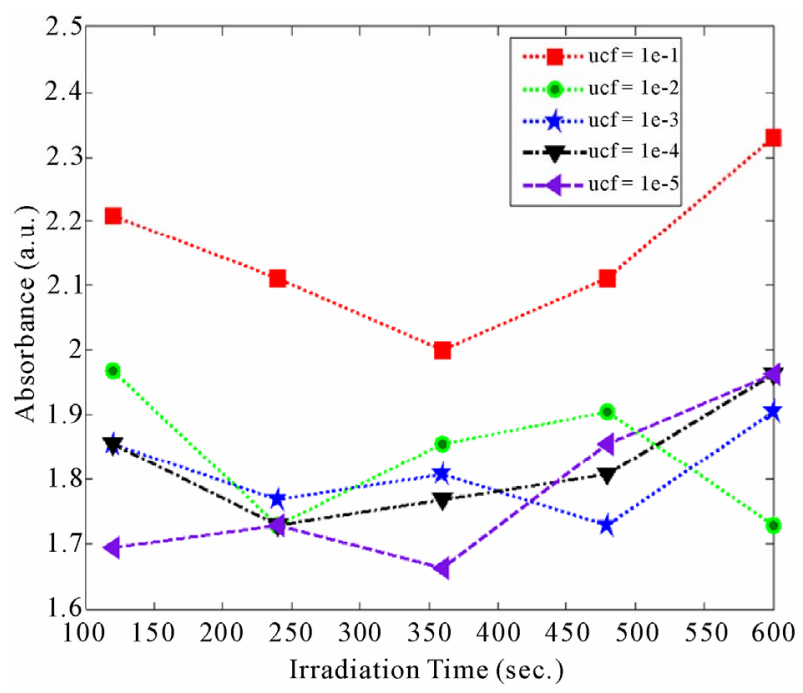

Figure 4. Variation of absorbance with the concentration for different irradiation time.

creasing irradiation time until certain irradiation time (depending on the sample concentration), stimulation effect is starting increasing with the increase of irradiation time. Moreover, there is an optimum concentration that exhibit best response as it is further illustrated in Figure 5.

In both figures the variation of absorbance with irradiation time for different concentration or the variation of absorbance with concentration for different irradiation time, it can be clearly noticed that there is significant variation for each case until certain specific irradiation time and/or concentration at which the trend of response is reversed. Meaning that by optimizing the aforesaid parameters, it is possible to control the required response. 


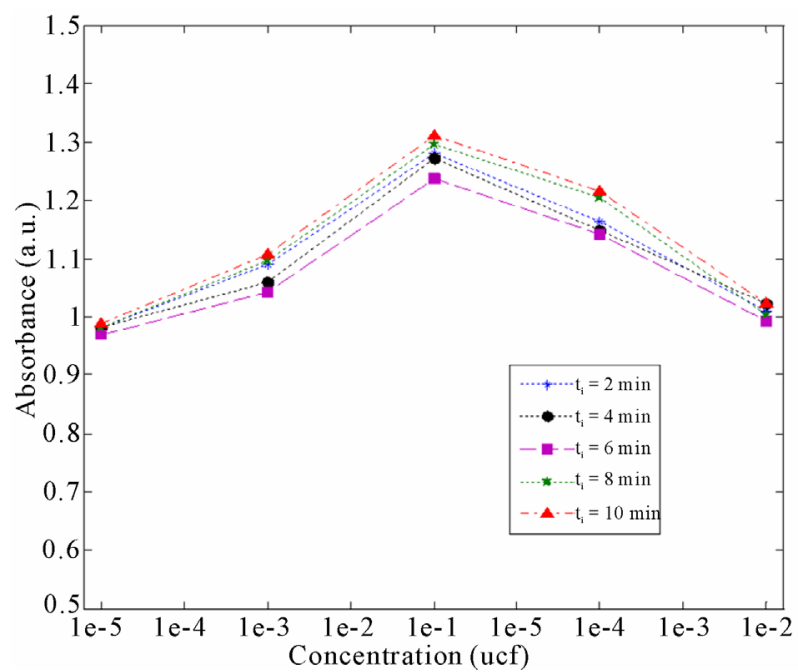

Figure 5. Variation of absorbance with the irradiation time for different concentration.

A linear variation of absorbance with the irradiation time was obtained when a laser power of $50 \mathrm{mw}$ was employed as shown in Figure 6. This means that there was a response resulted in an inhibition only without any stimulation which is manifested by the decrease of absorbance with the irradiation time. This suggests that low laser power can work better for wound healing in diabetes patients.

On the other hand, the scattered laser intensity from the irradiated sample has been also measured at the optimum irradiation time for different scattering angles as depicted in Figure 7.

There is an optimum scattering angle (50 degree) at which the scattered intensity is maximum and decreasing below and above that angle. The scattered intensity is also greatly affected by sample concentration and irradiation time (here the effect was best observed for irradiation time of $10 \mathrm{~min}$.). Moreover, the possibility of incorporating laser irradiation simultaneously with antibiotics as a therapeutic method for diabetic patients whose response to antibiotic is very slow was investigated with three different types of antibiotics, i.e., QB, $\mathrm{CB}$ and ZX and the result is illustrated in Figure 8. It is obvious that this procedure increases the impact effectiveness of the antibiotics on the investigated samples which is represented by the effective diameter range of the implanted samples.

All those results mean that good selection and optimization of the process parameters such as laser wavelength, intensity and the time of irradiation with the type of antibiotic is an important process to determine the effectiveness of the medical treatment utilizing these methods.

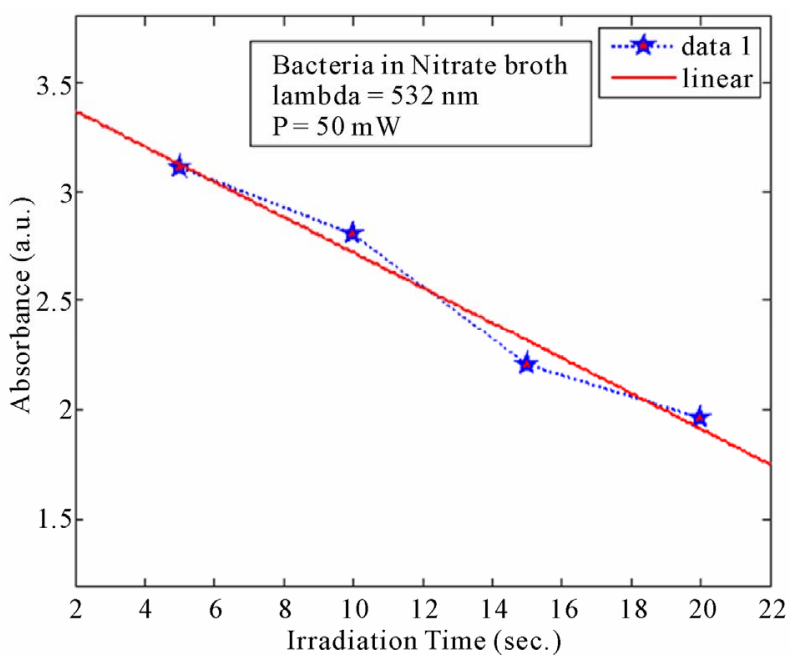

Figure 6. Variation of absorbance with the irradiation time for a $50 \mathrm{~mW}$ laser power.

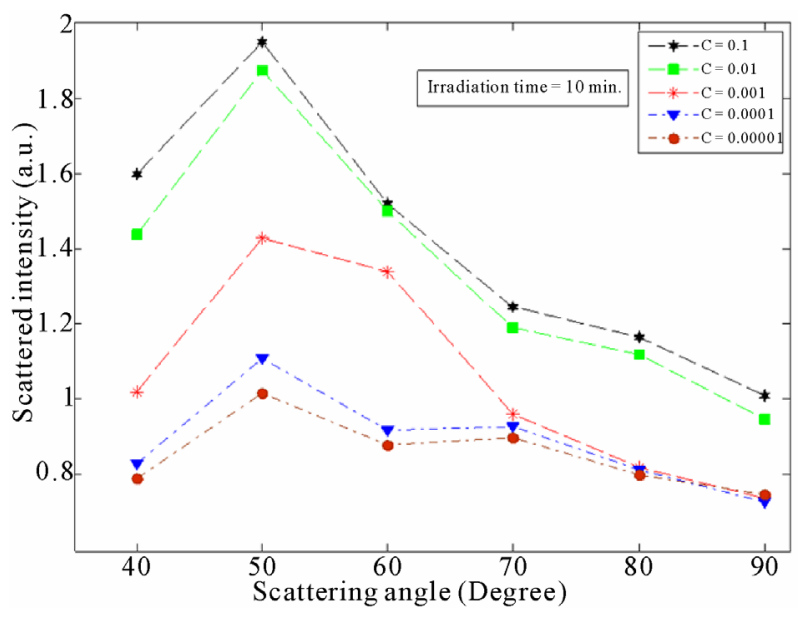

Figure 7. Variation of scattered intensity with the scattering angle for different concentrations.

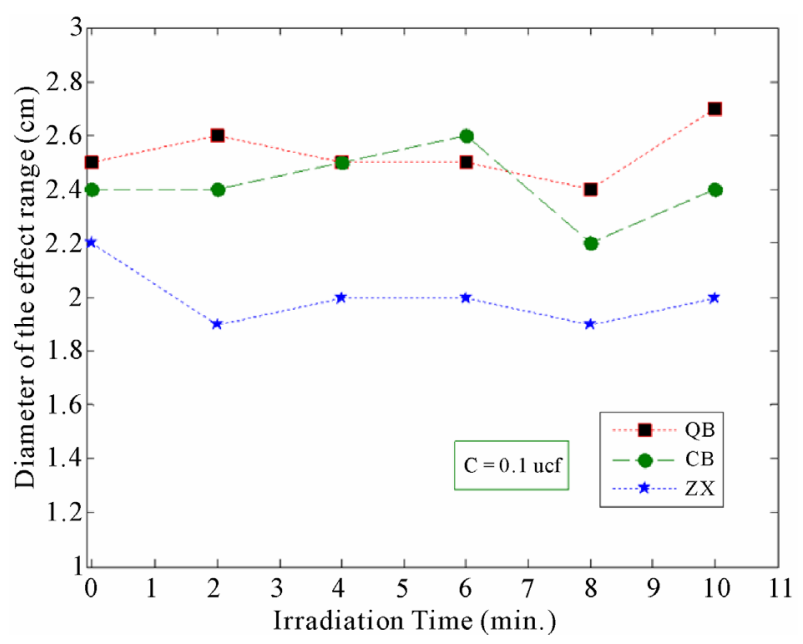

Figure 8. Variation of diameter of the effect range with irradiation time. 


\section{Conclusions}

Enzyme catalase and Staphylococcus Bacteria samples have been prepared in various concentrations and different conditions. They were irradiated for different irradiation times with different laser beam characteristics. From the obtained results, the photobiological stimulation and inhibition was clearly demonstrated for both Enzymes and Bacterial samples. That was clear from the absorbance and scattering trends. However, for the case of low laser power irradiation, it has been found that the irradiated bacterial samples experienced only inhibition effect which was obvious from the decreasing of absorbance with the irradiation time. Moreover, the simultaneous irradiation along with the anti-biotec incorporation shows that the effectiveness of the anti-biotec was significantly enhanced with the laser irradiation. The process of laser irradiation as well as optimization of both laser beam characteristics and samples conditions led to a conclusion of the effectiveness of laser irradiation on the investigated samples which means that this method with the required optimization can eventually be an effective therapeutic tool for many diseases especially for wound healing of diabetic patients. Moreover, further development of this technique can end up with an efficient tool for cancerous and malignant diseases.

\section{References}

[1] M. H. Niemz, "Laser-Tissue Interactions: Fundamentals and Applications," Springer-Verlag, Berlin, Heidelberg, 2004.

[2] M. H. Niemz, "Laser-Tissue Interactions: Fundamentals and Applications," Springer, Berlin, 1996, p. 297.

[3] K. C. Smith, "Light and Life: The Photobiological Basis of the Therapeutic Use of Radiation from Lasers," In: T. Oshiro and R. G. Calderhead, Eds., Progress in Laser Therapy Selected Papers from the 1st Meeting of the International Laser Therapy Association, Okinawa, 1990, pp. 11-18.

[4] T. Dai, B. Pikkula, V. Wang and B. Anvari, "Comparison of Human Skin Opto-Thermal Response to Near-Infrared and Visible Laser Irradiations: A Theoretical Investiga- tion,” Physics in Medicine and Biology, Vol. 49, No. 21, 2004, pp. 4861-4877. doi:10.1088/0031-9155/49/21/002

[5] T. Karu, "Derepression of the Genome after Irradiation of Human Lymphocytes with He-Ne Laser,” Laser Therapy, Vol. 4, 1992, pp. 5-24.

[6] H. Zheng, J. Qin, H. Yin and S. Yin, "The Activating Action of Low Level Helium Neon Laser Radiation on Macrophages in the Mouse Model,” Laser Therapy, Vol. 4, 1993, pp. 55-58.

[7] A. Loria and R. Kelly, "A Semiglobally Stable Output Feedback PI2D Regulator for Robot Manipulators,” IEEE Transactions on Automatic Control, Vol. 40, No. 8, 1995, pp. 1432-1436. doi:10.1109/9.402235

[8] A. S. Prasad, "Clinical and Biochemical Spectrum of Zinc Deficiency in Human Subjects,” In: A. S. Prasad, Ed., Clinical, Biochemical and Nutritional Aspects of Trace Elements, Alan R. Liss, Inc., New York, 1982.

[9] B. Kao, K. Kelly, G. Aguilar, Y. Hosaka, R. Barr and J. Nelson, "Evaluation of Cryogen Spray Cooling Exposure on in Vitro Model Human Skin," Lasers in Surgery and Medicine, Vol. 34, No. 2, 2004, pp. 146-154. doi:10.1002/lsm.10245

[10] R. Goepel, “Low Level Laser Therapy in France,” In: T. Oshiro and R. G. Calderhead, Eds., Progress in Laser Therapy. Selected Papers from the 1st meeting of the International Laser Therapy Association, Okinawa, 1990, pp. 71-74.

[11] C. Gartner, "Low Reactive-Level Laser Therapy (LLLT) in Rheumatology a Review of the Clinical Experience in the Author's Laboratory,” Laser Therapy, Vol. 4, No. 3, 1992, pp. 107-115.

[12] E. Wit and J. McClure, "Statistics for Microarrays: Design, Analysis, and Inference,” 5th Edition, John Wiley \& Sons Ltd., Chichester, 2004.

[13] K. Suthamjariya, W. Farinelli, W. Koh and R. Anderson, "Mechanisms of Microvascular Response to Laser Pulses,” Journal of Investigative Dermatology, Vol. 122, No. 2, 2004, pp. 518-525.

doi:10.1046/j.0022-202X.2004.22241.X

[14] S. LO and J. Nelson, "On the Physics of Laser-Induced Selective Photothermolysis of Hair Follicles: Influence of Wavelength, Pulse Duration, and Epidermal Cooling," Journal of Biomedical Optics, Vol. 9, No. 2, 2004, pp. 353-361. doi:10.1117/1.1646174 